\title{
In vivo production of pederin by labrenzin pathway expansion
}

\author{
Dina Kačar ${ }^{1}$, Carmen Schleissner ${ }^{2}$, Librada M. Cañedo ${ }^{2}$, Pilar Rodríguez ${ }^{2}$, Fernando de \\ la Calle ${ }^{2}$, Carmen Cuevas ${ }^{2}$, Beatriz Galán ${ }^{1}$ and José Luis García ${ }^{1}$ * \\ 1 Department of Microbial and Plant Biotechnology, Centro de Investigaciones \\ Biológicas, Agencia Estatal Consejo Superior de Investigaciones Científicas, Madrid, \\ Spain \\ 2 Research and Development Department, PharmaMar S.A., Madrid, Spain \\ *Corresponding author: jlgarcia@ cib.csic.es
}

\section{Summary}

Pederin is a potent polyketide toxin that causes severe skin lesions in humans after contact with insects of genus Paederus. Due to its potent anticancer activities, pederin family compounds have raised the interest of pharmaceutical industry. Despite extensive studies on the cluster of biosynthetic genes responsible for the production of pederin, it has not yet been possible to isolate and cultivate its bacterial endosymbiont producer. However, the marine bacterium Labrenzia sp. PHM005 was recently reported to produce labrenzin, the closest pederin analog. By cloning a synthetic pedO gene encoding one of the three $O$-methyltraferase of the pederin cluster into Labrenzia sp. PHM005 we have been able to produce pederin for the first time by fermentation in the new recombinant strain.

\section{Introduction}

Pederin, a natural polyketide, has a huge therapeutic potential as a highly potent anticancer agent (Richter et al., 1997), however up to now the only source of this compound was the insect Paederus fuscipes, where it is found in very low amounts since twenty-five million field-collected insects had to be used to isolate the minimal amount of pure pederin (Pavan and Bo, 1952) to determine its chemical structure (Cardani et al., 1965). Pederin is produced by one of the first trans-AT mixed type polyketide/non-ribosomal peptide synthases (PKS/NRPS) assigned to a natural product that has been identified in an as-yet uncultivated bacterial symbiont of the insect (Piel, 2002; Piel, Höfer, et al., 2004; Piel, Wen, et al., 2004) and thus, its production by bacterial fermentation has not been achieved yet. 
Pederin has among other structural peculiarities an $O$-methyl instead of the conventional ester or carboxylic acid polyketide terminus (Helfrich and Piel, 2010, 2016). In this sense, the cytotoxic activity of pederin-type compounds can be markedly increased by modifying the methylation pattern. The putative pederin biosynthetic gene cluster (ped) encodes three proteins with similarity to $O$-methyltransferases (MTs): PedA, PedE, and PedO (Piel, 2002; Piel, Hui, et al., 2004; Piel, Wen, et al., 2004). Biochemical in vitro experiments conducted with mycalamide as substrate, a pederin family compound with free $\mathrm{C} 18-\mathrm{OH}$ group, demonstrated that PedO was capable of introducing a methyl group at C18 position (Zimmermann et al., 2009).

Recently, the complete genome of the strain Labrenzia sp. PHM005, a freeliving and cultivable producer of a pederin analog 18-O-demethyl pederin (Schleissner et al., 2017; Benítez et al., 2021), hereinafter labrenzin, has been sequenced (Kačar et $a l ., 2019)$. A gene cluster responsible for the synthesis of labrenzin, named lab cluster, has been identified showing that it encodes a trans-AT mixed type PKS/NRPS biosynthetic pathway (Kačar et al., 2019). Interestingly, the lab cluster only encodes two MTs and this observation suggested that Labrenzia sp. PHM005 could be a good chassis to biosynthetically produce pederin by engineering the missing MT tailoring gene of ped cluster.

The aim of this work was to demonstrate that pederin could be produced by fermentation in Labrenzia sp. PHM005 by the heterologous expression of a pedO synthetic gene. In addition, further improvements of the pederin production have been investigated by overexpressing other MTs.

\section{Materials and methods}

Bacterial strains, media and growth conditions

Standard overnight Escherichia coli MFDpir (Ferrières et al., 2010) cultures were grown aerobically in Luria-Bertani (LB) broth or LB agar at $37^{\circ} \mathrm{C}$ (Bertani, 1951). The medium was supplemented with $1 \mathrm{mM}$ diamine-pymelic acid (DAP) and the corresponding antibiotic, when appropriate. Labrenzia sp. PHM005 wild type and recombinant strains were grown in Marine Broth (MB) Difco 2216 (Sigma-Aldrich) or Marine Agar (MA) Difco 2216 (Sigma-Aldrich), supplemented with antibiotics, when appropriate. All the strains were cultured in $50 \mathrm{~mL}$ falcon tubes or $100 \mathrm{~mL}$ flasks with 10 and $20 \mathrm{~mL}$ of medium, respectively. Culture medium used to study labrenzin and pederin production in Labrenzia sp. PHM005 was modified using marine basal medium 
supplemented with vitamins (MBM+vit) (Kačar et al., 2019). The culture medium was supplemented with $0.2 \mathrm{mM} 3$-methyl-benzoate, when the cloned gene was expressed under the control of the inducible $P m$ promoter. The strains were grown overnight in falcon tubes in $\mathrm{MB}$ at $30^{\circ} \mathrm{C}$ with shaking at $200 \mathrm{rpm}$. The overnight culture was washed in $0.85 \% \mathrm{NaCl}$ solution and diluted to an optical density $\left(\mathrm{OD}_{600}\right) \approx 0.1$ in $20 \mathrm{~mL}$ of fresh medium. To determine the production of labrenzin, pederin and analogs by HPLC/MS analyses, the strains were cultured for $72 \mathrm{~h}$.

\section{Plasmid DNA transformation and clone selection}

E. coli MFDpir electro-competent cells were prepared and transformed by electroporation as described (Wirth et al., 1989). To select the transformants chloramphenicol $(34 \mu \mathrm{g} / \mathrm{mL})$ and $1 \mathrm{mM}$ DAP was added to the LB agar plates. A biparental conjugation was used for transformation of Labrenzia sp. PHM005 using E. coli MFDpir carrying the plasmid of interest as a donor strain. Samples of $1 \mathrm{~mL}$ of overnight cultures of Labrenzia and E. coli MFDpir were collected by centrifugation and the pellets were washed with $500 \mu \mathrm{L}$ of $0.85 \% \mathrm{NaCl}$ and resuspended into $200 \mu \mathrm{L}$ of the same solution. Samples of $50 \mu \mathrm{L}$ of each strain were mixed and deposited into a $0.22 \mu \mathrm{L}$ filter mating disc placed on the surface of a MA agar plate that was further incubated for 4-6 h at $37^{\circ} \mathrm{C}$. The cells deposited on the filter disc were collected with 1 $\mathrm{mL}$ of $0.85 \% \mathrm{NaCl}$ and vortexed thoroughly to detach the cells from the filter. Afterwards, cells were plated by dilutions on MA plates containing chloramphenicol (5 $\mu \mathrm{g} / \mathrm{mL})$.

\section{Construction of plasmids for expression of MTs}

Plasmid pSEVA338 from pSEVA collection (http://seva-plasmids.com/) was used as vector to create an artificial operon containing three genes encoding the Lab6 and Lab16 MTs from Labrenzia and PedO MT from Paederus symbiont. The artificial operon was designed as indicated in Figure S1, where each gene with the corresponding RBS was flanked by specific restriction sites (blunt cut) in a way that individual genes or the combination of genes could be easily generated by digestion and re-ligation. The operon was synthetized and cloned using SacI and SpeI restriction sites by GenScript yielding plasmid pSEVA338_MTs. Other plasmids derived from pSEVA338_MTs were pSEVA338_lab6, pSEVA338_lab16, pSEVA338_pedO, pSEVA338_lab6_lab16, pSEVA338_lab6_pedO and pSEVA338_lab16_pedO. The inducible promoter Pm from 
pSEVA338 was replaced by the strong $p 14 g$ promoter in plasmid pSEVA227M (kind gift from Gonzalo Durante) using restriction enzymes PacI and AvrII generating a new construct pSEVA338_p14g_lab6_pedO.

\section{Extraction, purification and identification of polyketide compounds}

Upon fermentation $20 \mathrm{~mL}$ of culture medium collected by centrifugation was frozen at $80{ }^{\circ} \mathrm{C}$ and subsequently freeze dried. The lyophilized product was then dissolved in 4 $\mathrm{mL}$ of distilled water and equal volume of ethyl acetate. After homogenization and centrifugation, the organic phase was collected by pipetting and the extraction was repeated once more. The collected organic phase was dried by vacuum centrifugation and the pellet was dissolved in $150 \mu \mathrm{L}$ of methanol and filtered for a HPLC/MS analysis.

HPLC-MS analysis was carried out using a HPLC/MS system and a separation column previously described (Kačar et al., 2019). The running method was as follows: solvent A was $100 \%$ water and solvent B was $100 \%$ acetonitrile. The flow rate was $500 \mu \mathrm{L}$ $\min ^{-1}$ using the following gradient: $\mathrm{t}=2 \min , 100 \% \mathrm{~A} ; \mathrm{t}=8 \min , 95 \% \mathrm{~A} ; \mathrm{t}=40 \min$, $55 \% \mathrm{~A} ; \mathrm{t}=53 \min , 0 \% \mathrm{~A} ; \mathrm{t}=55 \mathrm{~min}, 0 \% \mathrm{~A} ; \mathrm{t}=57 \mathrm{~min}, 100 \% \mathrm{~A} ; \mathrm{t}=65 \mathrm{~min}, 100 \% \mathrm{~A}$.

\section{Results}

\section{Genetic analyses}

The unique structural difference between pederin and labrenzin is the absence of an $O$ methylation of $\mathrm{C} 18-\mathrm{OH}$ in labrenzin (Figure 1). The absence of such methylation can be justified assuming that the lab cluster contains only two MTs, i.e., Lab6 (MT6) and Lab16 (MT16) (Kačar et al., 2019), whereas ped cluster contains three MTs, i.e., PedA, PedE and PedO. A protein homology comparison between MTs from lab and ped clusters showed that Lab16 is homologous to PedE with a 51\% amino acid sequence identity and Lab6 shares $47 \%$ sequence identity with PedA. According to Blast analysis the sequence amino acid identity of PedO with Lab16 was very low (28\% with a query cover of 63\%) but Lab6 and PedO showed a 53\% sequence identity. Although the genetic analysis cannot exclude Lab6 from the experimental setting, the previous in vitro biochemical analysis carried out with PedO using mycalamide A as substrate demonstrated that PedO is responsible for the methylation of the $\mathrm{C} 18-\mathrm{OH}$ group in pederin (Zimmermann et al., 2009). Thus, we assumed that PedO could be the missing gene in Labrenzia responsible of the $\mathrm{C} 18-\mathrm{OH}$ methylation in the bacterial symbiont. 
Nevertheless, considering that PedO methylation activity was only tested in vitro using mycalamide as substrate our hypothesis required an experimental in vivo demonstration since PedO could not methylate labrenzin under the in vivo environmental conditions where the concentrations of the reaction substrates inside the cells are different than those used for the methylation of mycalamide in vitro and different labrenzin intermediates could compete as putative substrates or even work as inhibitors. Moreover, although PedO was produced in an active form in Escherichia coli after the optimization of its expression (Zimmermann et al., 2009), the gene and/or the enzyme could require some additional optimization to become active in Labrenzia.

\section{Production of pederin in Labrenzia sp. PHM005}

To test our hypothesis, this is, to be able to synthetize pederin for the first time in a cultivable bacterium, a synthetic pedO gene was heterologously expressed in Labrenzia sp. PHM005 transformed with the recombinant plasmid pSEVA338_pedO harbouring the ped $O$ synthetic gene under the control of the $P_{m}$ inducible promoter. As predicted, when a culture extract of Labrenzia sp. PHM005 (pSEVA338_pedO) was analyzed by HPLC/MS we observed a new intermediate, more hydrophobic than the previous pederin analogs, i.e., compound (1) (labrenzin) and compound (2) (17-O-demethylated labrenzin) produced by the wild type strain (Figure 2). The MS spectrum revealed a new peak (compound 3) observed at $\mathrm{RT}=40.4 \mathrm{~min}$ and with ion fragmentation $\mathrm{m} / \mathrm{z}=526$; $440 ; 422$. This fragmentation pattern is matching with both labrenzin and 17-Odemethylated labrenzin (Figure S2) and indicates an additional methyl group (M+14), as it is in pederin.

\section{Co-overexpression of lab6, lab16 and PedO methylases}

Although pederin was produced in Labrenzia sp. PHM005 (pSEVA338_pedO), it was not the most abundant intermediate in the extract. Therefore, to optimize the pederin production we analysed the production in different strains co-expressing the pedO gene in different combinations with lab6 and lab16. To this aim, plasmids pSEVA338_lab6_pedO, pSEVA338_lab16_pedO, pSEVA338_lab6_lab16_pedO were constructed and transformed in Labrenzia sp. PHM005. The resulting strains were cultivated in the production medium and the production of the labrenzin analogues was analysed. Figure 3 (A-E) shows HPLC-MS chromatograms of culture extracts of strains harnessing different expression combinations of MTs using pSEVA338 plasmid. The 
first conclusion was that the relative pederin amount was increased by co-expressing pedO with lab6 and labl6 genes in the different combinations tested (Figure 3E and 3F). 17-O-demethylated labrenzin (compound 2) was the most abundant peak when ped $O$ was expressed alone forming an operon with labl6 and when the three MTs encoding genes were co-expressed (Figure 3C and 3D). However, ped $O$ overexpressed together with lab6, appeared to be the optimal combination for pederin production under the culture conditions.

\section{Engineering the promoter driven the expression of the lab6_pedO MTs}

The second approach was to engineer the expression plasmid pSEVA338_lab6_pedO with the constitutive strong $P_{14 g}$ promoter, replacing the inducible $P_{m}$ promoter. Previously, we tested the $P_{14 g}$ promoter strength in Labrenzia sp. PHM005 by constructing a transcriptional fusion with GFP showing that it is functional and has high expression levels (data not shown). The resulting plasmid named pSEVA338_p14g_lab6_pedO was transformed in Labrenzia sp. PHM005. The production of the recombinant strain was analysed showing that the promoter switch did not seem to alter the peak ratio as observed in the MS chromatograms (Figure 3F), suggesting that the expression of pedO is not the bottleneck of the process.

\section{Discussion}

$O$-Methylation modulates the pharmacokinetic and pharmacodynamic properties of natural products, affecting their bioavailability, stability, and binding to targets. Tailoring the polyketide structures allows an additional level of functional complexity, and thus, polyketide pathway engineering has generated new-to-nature products through novel glycosylation, acyltransferase, hydroxylation, epoxidation, alkylation, transamination and desaturation reactions acting on naturally occurring products (Cumming et al., 2014). However, as far as we know, very few experiments have been carried out to expand a polyketide pathway to generate novel polyketides by cloning tailoring $O$-MTs. Many years ago, Fu et al. (1996) expressed the TcmO $O$-MT of the tetracenomycin biosynthetic pathway of Streptomyces glaucescens was expressed in Streptomyces coelicolor $\mathrm{CH} 999$ together with the actinorhodin polyketide synthase (PKS) gene cluster, which is responsible for the biosynthesis of 3,8-dihydroxymethylanthraquinone carboxylic acid (DMAC) and its decarboxylated analog, aloesaponarin. The resulting recombinant strain produced approximately equal 
quantities of aloesaponarin and a new product but no DMAC. More recently, Wang et al. (2019) have studied the use of two fungal MTs to produce unnatural O-methylated benzenediol lactone polyketides.

In this work, we have confirmed the role of PedO MT found in Paederus bacterial symbiont. In addition, we have demonstrated that its heterologous expression in recombinant strains of Labrenzia sp. PHM005 has allowed expanding the labrenzin biosynthetic pathway generating a new compound, pederin, providing labrenzin with an additional methylation on $\mathrm{C} 18-\mathrm{OH}$. As mentioned above, pederin can be only isolated from beetle extraction so far, and thus, this is the first time pederin is produced by direct fermentation in a cultivable bacterium. Therefore, pederin could be now produced by fermentation at large scale to be tested and used as an antitumoral drug. Nevertheless, although we have developed different ped $O$ expression systems for the production of pederin further improvements should be made for its efficient industrial scale production since the production levels of labrenzin and pederin are still low. In addition to increasing production of the labrenzin polyketide scaffold, we have shown that a finetuning of the expression of lab6 and pedO genes should be optimized to increase the pederin production. It is possible that sequential $O$-methylation tailoring would depend on the substrate preference of MTs resulting the synthesis of the intermediates more favorable than that of pederin. A similar finding was observed when PedO was unable to methylate the $\mathrm{C} 18-\mathrm{OH}$ group of mycalamide A intermediate when the neighboring $\mathrm{OH}$ was methylated, i.e., C18 $\mathrm{O}$-methylation appeared only possible when the $\mathrm{C} 17-\mathrm{OH}$ was non methylated (Zimmermann et al., 2009). On the other hand, the synthetic pedO gene used in this study originates from an uncultured symbiont bacterium (Piel et al., 2005), therefore, its expression might not be very efficient in Labrenzia sp. PHM005. In that sense, for more efficient pederin transformation from labrenzin in the industrial setting, we suggest to carry out codon usage modifications or to approach a directed evolution of PedO enzyme. Finally, it is important to consider that the production of a combination of different sequentially methylated intermediates, all of them secreted to the surrounding medium, can provide an advantage for the producing bacteria, since different products can develop different roles in their surrounding environment. Thus, the simultaneous production and secretion of the intermediates can be detrimental for the industrial overproduction of pederin, since we have determined that labrenzin once secreted to the medium cannot be uptake by the cells (data not shown) and therefore, it cannot be methylated. 


\section{Acknowledgements}

The present study was funded by the Ministry of Economy and Competitiveness of Spain under the program RETOS-COLABORACIÓN with the project number RTC2016-4892-1 (DESPOL). The authors would like to thank Ana Valencia for technical assistance.

\section{References}

Benítez, X., González, E.G., García, J., Zúñiga, P., de la Calle, F. and Cuevas C. (2021) Detection of a pederin-like compound using a dilution-to-extinction-based platform for the isolation of marine bacteria in drug discovery strategies. Microb Biotechnol 14: 241-250.

Bertani, G. (1951) Studies on lysogenesis. I. The mode of phage liberation by lysogenic Escherichia coli. J Bacteriol 62: 293-300.

Cardani, C., Ghiringhelli, D., Mondelli, R., and Quilico, A (1965) The structure of pederin. Tetrahedron Lett 6: 2537-2545.

Cummings, M., Breitling, R. and Takano, E. (2014) Steps towards the synthetic biology of polyketide biosynthesis. FEMS Microbiol Lett 351: 116-125.

Ferrières, L., Hémery, G., Nham, T., Guérout, A.M., Mazel, D., Beloin, C. and Ghigo, J.M. (2010) Silent mischief: bacteriophage Mu insertions contaminate products of Escherichia coli random mutagenesis performed using suicidal transposon delivery plasmids mobilized by broad-host-range RP4 conjugative machinery. J Bacteriol 192: 6418-6427.

Fu, H., Alvarez, M.A., Khosla, C. and Bailey, J.E. (1996) Engineered biosynthesis of novel polyketides: regiospecific methylation of an unnatural substrate by the tcmO O-methyltransferase. Biochemistry 35: 6527-6532.

Helfrich, E.J.N. and Piel, J. (2010) Biosynthesis of polyketides by trans-AT polyketide synthases. Nat Prod Rep 27: 996-1047.

Helfrich, E.J.N. and Piel, J. (2016) Biosynthesis of polyketides by trans-AT polyketide synthases. Nat Prod Rep 33: 231-316.

Kačar, D., Schleissner, C., Cañedo, L.M., Rodríguez, P., Calle, F. De, Galán, B. and García, J.L. (2019) Genome of Labrenzia sp. PHM005 reveals a complete and active trans-AT PKS gene cluster for the biosynthesis of labrenzin. Front Microbiol 10: 1-14. 
Pavan, M. and Bo, G. (1952) Ricerche sulla differenziabilita, natura e attivita del principio tossico di Paederus fuscipes Curt.(Col. Staph.). Mem Soc Ent It 67-82.

Piel, J. (2002) A polyketide synthase-peptide synthetase gene cluster from an uncultured bacterial symbiont of Paederus beetles. Proc Natl Acad Sci U S A 99: 1400214007.

Piel, J., Butzke, D., Fusetani, N., Hui, D., Platzer, M., Wen, G. and Matsunaga, S. (2005) Exploring the chemistry of uncultivated bacterial symbionts: Antitumor polyketides of the Pederin family. J Nat Prod 68: 472-479.

Piel, J., Höfer, I. and Hui, D. (2004) Evidence for a symbiosis island involved in horizontal acquisition of pederin biosynthetic capabilities by the bacterial symbiont of Paederus fuscipes beetles. 186: 1280-1286.

Piel, J., Hui, D., Fusetani, N. and Matsunaga, S. (2004) Targeting modular polyketide synthases with iteratively acting acyltransferases from metagenomes of uncultured bacterial consortia. Environ Microbiol 6: 921-927.

Piel, J., Wen, G., Platzer, M. and Hui, D. (2004) Unprecedented diversity of catalytic domains in the first four modules of the putative pederin polyketide synthase. ChemBioChem 5: 93-98.

Richter, A., Kocienski, P., Raubo, P. and Davies, D. (1997) The in vitro biological activities of synthetic 18-O-methyl mycalamide B, 10-epi-18-O-methyl mycalamide B and pederin. Anticancer Drug Des 12: 217-227(11).

Schleissner, C., Cañedo, L.M., Rodríguez, P., Crespo, C., Zúñiga, P, Peñalver, A., de la Calle, F. and Cuevas, C. (2017) Bacterial production of a pederin analogue by a free-living marine Alphaproteobacterium. J Nat Prod 80: 2170-2173.

Wang, X., Wang, C., Duan, L., Zhang, L., Liu, H., Xu, Y.M., Liu, Q., Mao, T., Zhang, W., Chen, M., Lin, M., Gunatilaka, A.A.L., Xu, Y. and Molnár, I. (2019) Rational reprogramming of O-methylation regioselectivity for combinatorial biosynthetic tailoring of benzenediol lactone scaffolds. J Am Chem Soc 141: 4355-4364.

Wirth, R., Friesenegger, A. and Fiedler, S. (1989) Transformation of various species of gram-negative bacteria belonging to 11 different genera by electroporation. $M G G$ Mol Gen Genet 216: 175-177.

Zimmermann, K., Engeser, M., Blunt, J.W., Munro, M.H.G. and Piel, J. (2009) Pederintype pathways of uncultivated bacterial symbionts: Analysis of $\mathrm{O}$ methyltransferases and generation of a biosynthetic hybrid. J Am Chem Soc 131: 2780-2781. 
bioRxiv preprint doi: https://doi.org/10.1101/2021.09.29.462382; this version posted October 1, 2021. The copyright holder for this preprint (which was not certified by peer review) is the author/funder, who has granted bioRxiv a license to display the preprint in perpetuity. It is made available under aCC-BY-NC-ND 4.0 International license. 


\section{Legends to the figures}

Figure 1. Chemical structure of pederin family polyketides.

Figure 2. Pederin biosynthesis in Labrenzia sp. PHM005 wt (pSEVA338_pedO). A) HPLC-MS chromatograms of the supernatant extracts obtained after $72 \mathrm{~h}$ of cultivation of wt cultures in MBM+vit medium presenting extracted ions in the range $\mathrm{m} / \mathrm{z}=498$ 526. Intermediates compound (1) (labrenzin), compound (2) (17-O-demethylated labrenzin) and compound (3) (pederin) are indicated. B) ESIMS ion fragmentation of compound (3) (pederin).

Figure 3. HPLC-MS chromatograms of the culture extracts obtained after $72 \mathrm{~h}$ of cultivation of wt recombinant strains transformed with different plasmids in MBM+vit medium. Extracted ions range is $\mathrm{m} / \mathrm{z}=498-526$. Intermediates $1(\mathrm{~m} / \mathrm{z}=512), 2(\mathrm{~m} / \mathrm{z}=$ $498)$ and $3(\mathrm{~m} / \mathrm{z}=526)$ are indicated. A) wt; B) wt(pSEVA338_pedO); C) wt(pSEVA338_lab6_lab16_pedO); D) wt(pSEVA338_lab16_pedO); E) wt(pSEVA338_lab6_pedO); F) wt(pSEVA338_p14g_lab6_pedO).

Figure S1. Scheme representing the design of the artificial operon comprising PedOLab6-Lab16 methyltransferases, under the control of the Pm promoter of pSEVA338.

Figure S2. ESIMS ionization of A) labrenzin and B) 17-O-demethyl labrenzin. 

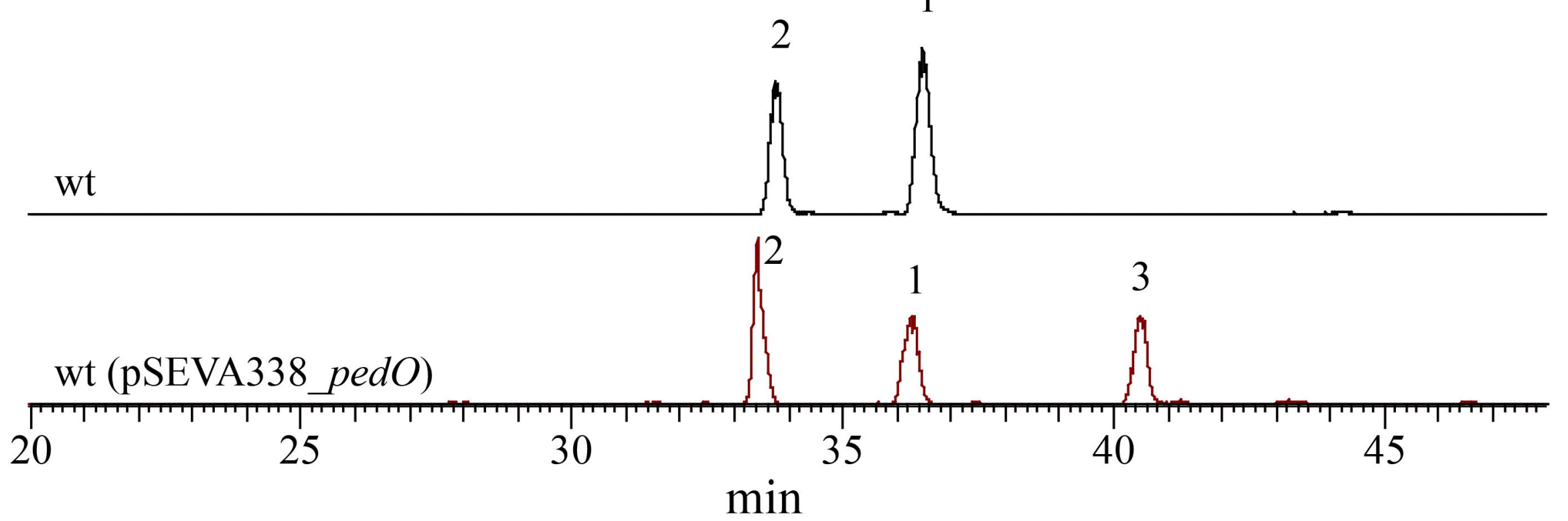

B

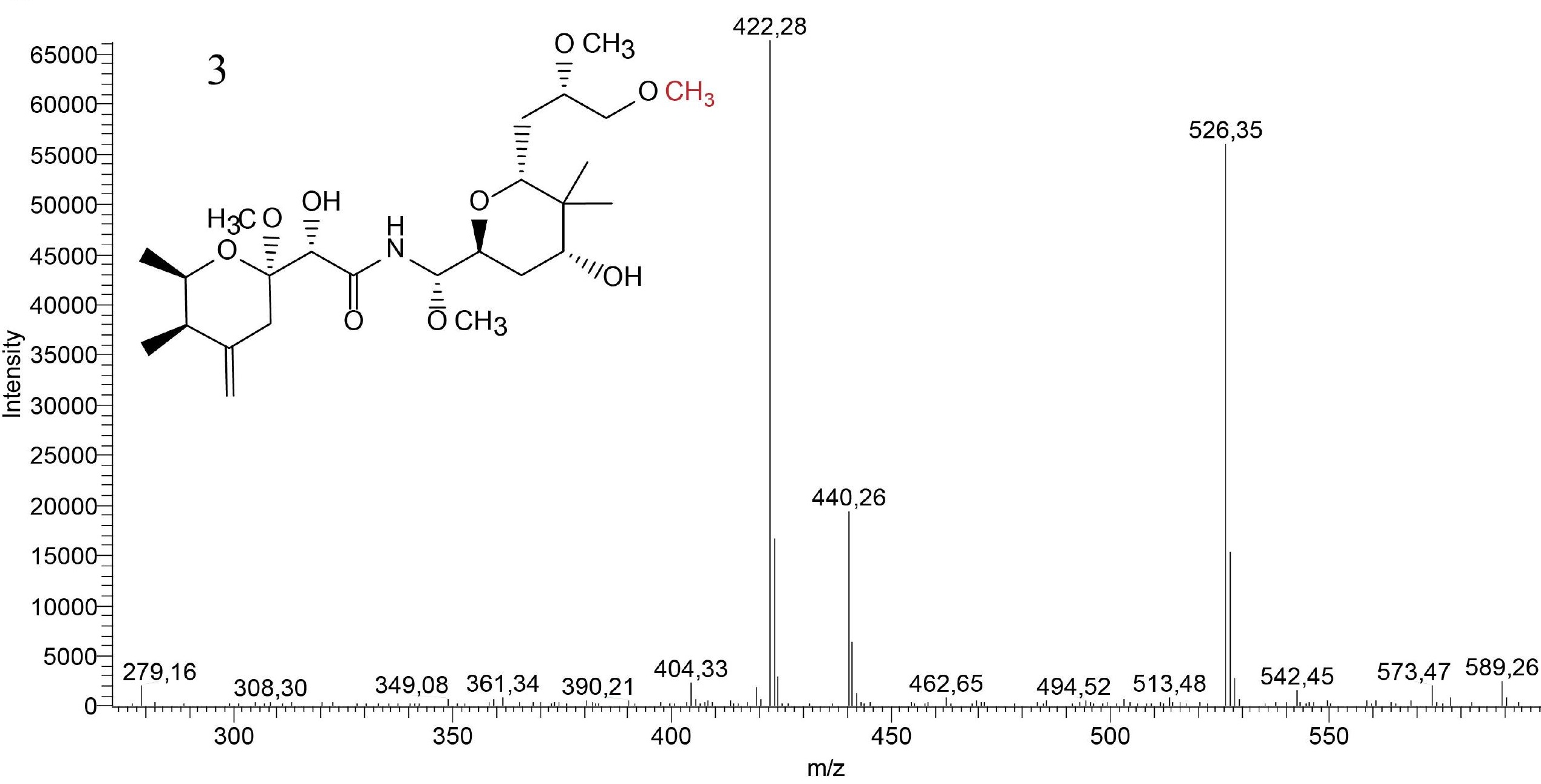


B

wt (pSEVA338_pedO)

C

wt (pSEVA338_lab6_lab16_pedO)

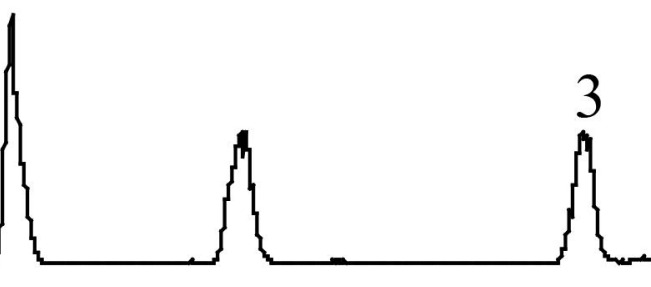

D

wt (pSEVA338_lab16_pedO)

E

wt (pSEVA338_lab6_pedO)

F

wt (pSEVA338_p14g_lab6_pedO) 